\title{
Secondary-electron emission from hydrogen-terminated diamond: Experiments and model
}

\author{
Erdong Wang, ${ }^{1}$ Ilan Ben-Zvi, ${ }^{2,3}$ Triveni Rao, ${ }^{4}$ D. A. Dimitrov, ${ }^{5}$ Xiangyun Chang, ${ }^{2}$ Qiong Wu, ${ }^{2}$ and Tianmu Xin ${ }^{3}$ \\ ${ }^{1}$ State Key Laboratory of Nuclear Physics and Technology, Institute of Heavy Ion Physics, School of Physics, \\ Peking University, Beijing 100081, China \\ ${ }^{2}$ Collider Accelerator Department, Brookhaven National Laboratory, Upton, New York 11973, USA \\ ${ }^{3}$ Physics and Astronomy Department, Stony Brook University, Stony Brook, New York 11974, USA \\ ${ }^{4}$ Instrumentation Division, Brookhaven National Laboratory, Upton, New York 11973, USA \\ ${ }^{5}$ Tech-X Corp., Boulder, Colorado 80303, USA \\ (Received 1 August 2011; published 23 November 2011)
}

\begin{abstract}
A hydrogen-terminated diamond is a proven efficient electron emitter that can support emission of high average current. Several factors dictate the amplifier's gain: the number of secondary electrons created at their point of entry into the diamond, the fraction of created electrons transmitted to the emitting face, and the fraction of transmitted electrons emitted. In this paper, we present a model detailing the impact of charge trapping at the surface on the instantaneous electric field inside the diamond, and its effect on the transmission gain. The ratio of instantaneous emitted electrons to the transmitted electrons depends on the electron's energy distribution and the surface barrier. We calculated the latter by evaluating the magnitude of the negative-electron affinity that is modified by the Schottky effect due to the presence of the external applied field. The instantaneous values then were time integrated to yield the time-averaged ratio of the number of emitted electrons to the transmitted ones. The findings from the model agree very well with our experimental measurements. As an application of the model, we estimate the energy spread of the electrons inside the diamond from the measured secondary-electron emission.
\end{abstract}

DOI: 10.1103/PhysRevSTAB.14.111301

PACS numbers: 29.25.Bx

\section{INTRODUCTION}

New light sources require an electron source with high average current and high brightness. Diamond amplifiers demonstrably are an electron source with the potential to support high-brightness, high-average-current emission into a vacuum. In previous experiments, we demonstrated an emission gain up to 178 [1].

Briefly, a diamond amplifier functions as follows. Primary electrons with energy of a few $\mathrm{keV}$ enter a highpurity single-crystal diamond through a metal coating. These electrons excite electron-hole pairs, the number of which typically is about 2 orders of magnitude more than the number of primary electrons, depending on the latter's energy. A fraction of the electrons is lost to recombination near the diamond-metal interface and by diffusion into the metal surface. The remaining secondary electrons drift across the diamond under the influence of an externally applied, pulsed rectangular longitudinal shape electric field generated by a push-pull high voltage (HV) switch circuit and reach the hydrogen-terminated surface. Of those reaching this surface, a fraction of them is emitted; the rest are trapped there, and accumulate until the external field is shielded completely. Hence, the field inside the

Published by the American Physical Society under the terms of the Creative Commons Attribution 3.0 License. Further distribution of this work must maintain attribution to the author(s) and the published article's title, journal citation, and DOI. diamond changes with time, resulting in a transmitted charge, and thereby an emitted charge, both of which are time dependent. Our previous experiments show that this shielding takes place over $\sim 1 \mu \mathrm{s}$, a relatively slow process [2]. It is important to assess precisely the probability of the emission of an electron that reaches the emission surface, and to understand the mechanism responsible for its trapping and its dependence on external conditions. We approached the problem as follows: We prepared four nearly identical hydrogenated-diamond samples following the procedure outlined in Ref. [1] and measured the fraction of electrons emitted as a function of the pulse length and magnitude of the applied field. We propose a model of emission that rests upon the measured effect of the shielding on recombination near the metal-diamond interface.

\section{DERIVATION OF THE TIME-DEPENDENT INTERNAL FIELD}

The primary current, the primary electrons' energy, and the internal electric field determine the secondary-electron current reaching the emission surface. In the following section, we describe the model we developed to calculate the changes in the fields inside the diamond, and on the emission surface as a function of time due to the trapping of electrons, along with the impact of these changes on the fraction of the electrons emitted after arriving at the diamond's emission surface. We derived the time-dependent field inside the diamond as a function of instantaneous 
transmission gain and the emission probability. The transmission gain was determined from earlier experimental data. The emission probability depends on the distribution of electron energy, and the surface potential barrier, which can be modified by the applied field and the field due to the image charges inside the diamond. The energy distribution and the surface barrier were calculated from the emission data from four samples. The time-dependent field, thus derived, was used to determine the instantaneous emission current. The integrated emission current and average gain then was compared to the experimental results.

We start by defining various variables (Fig. 1), wherein $I_{p}$ is the DC primary current, and the generated average secondary-electron current is $I_{s}$. The instantaneous secondary-electron current reaching the emission surface is $I_{i}(t)$. The instantaneous current emitted from the diamond is defined as $I_{e}(t)$. The measured average emission secondary-electron current is $I_{a}=\left(\int_{0}^{w} I_{e}(t) d t\right) /(1 / f)$, where $f$ is the pulse repetition frequency and $w$ is the high-voltage pulse width. The instantaneous transmission gain is defined as $G_{t}=\frac{I_{i}}{I_{p}}$. We further define the average emission gain as $G_{e}=\frac{I_{a}}{I_{p}} \frac{1}{f \cdot w}$. We want to extract from the measurements of the average emission current $I_{a}$ as a function of applied field and pulse length the instantaneous emission probability, defined as $P=I_{e} / I_{i}$. In a very short high-voltage pulse width $(\ll \mu \mathrm{s})$, when the density of the surface-trapped electrons is insufficient to shield the external field, the emission probability is equal to $P=$ $I_{a} /\left(I_{i} \cdot w \cdot f\right)$ or equivalently $P=G_{e} / G_{t}$.

Based on these definitions, we can write the timedependent current of secondary electrons reaching the emission surface as

$$
I_{i}(t)=I_{p} \times G_{t}\left[\mathcal{E}_{p}, E_{i}(t)\right],
$$

where the transmission gain is a function of the energy of the primary electrons $\mathcal{E}_{p}$, and the diamond's internal

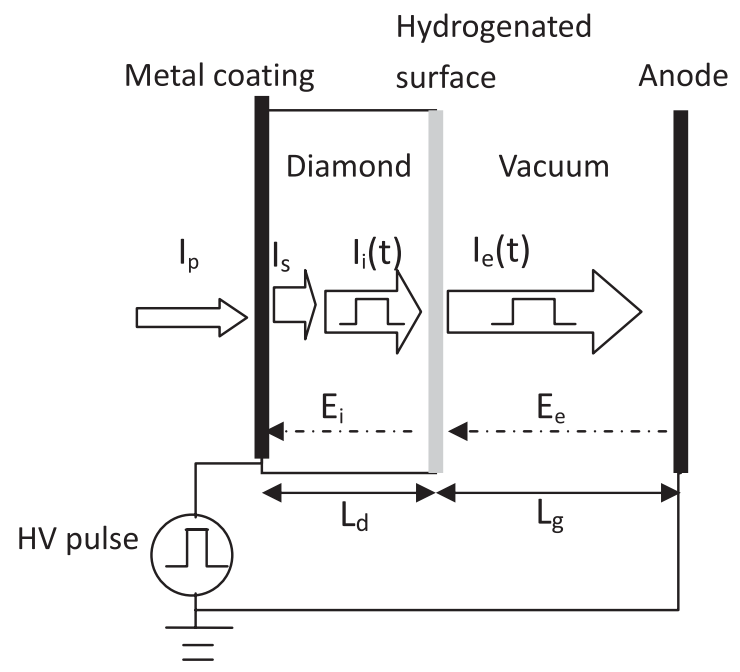

FIG. 1. The definitions of currents and fields in the diamond amplifier. electric field, $E_{i}$. The time dependence is induced by the variations in the internal electric field $E_{i}(t)$ due to shielding by the trapped charge. The density of trapped electrons is given by

$$
\sigma(t)=\frac{1}{S} \int_{0}^{t}(1-P) I_{i}(t) d t,
$$

where the $S$ is the size of the secondary-electron spot. Diamond amplifiers are drift-dominated cathodes, so the diameter of secondary-electron beam reaching the emission surface of a thin diamond essentially is the same as that of the primary bunch, which we can measured experimentally. $P$ is the emission probability, defined above.

The internal field is the vector sum of the external field and the field induced by the density of trapped electrons. For calculating the internal field in the surface-trapped diamond amplifier, we used the capacitor model. The internal field is given by

$$
E_{i}(t)=\frac{E_{e}}{\varepsilon_{r}}-\frac{\sigma(t)}{\varepsilon},
$$

where $E_{e} / \varepsilon_{r}$ is the internal field without surface trapping, $\varepsilon_{r}$ is the relative permittivity of pure diamond, and $\varepsilon$ is the permittivity of the diamond. In our emission test setup, we applied a constant high-voltage pulse to the diamond's metal coating, and the anode was grounded. The external field increases when the emission surface accumulates trapped secondary electrons. The external field is expressed by

$$
E_{e}(t)=\frac{V-E_{i}(t) \times L_{d}}{L_{g}},
$$

where $V$ is the high voltage on the diamond's metal coating. $L_{d}$ is the thickness of the diamond, and the $L_{g}$ is the distance between the diamond's emission surface and the anode. The surface electron trapping and the reduction in the internal field are described by

$$
E_{i}(t)=\frac{E_{e}}{\varepsilon_{r}}-\frac{1}{\varepsilon \times S} \int_{0}^{t}(1-P) \times I_{p} \times G_{t}\left[E_{p}, E_{i}(t)\right] d t .
$$

To solve this integral equation, we must know the amplifier's transmission gain $G_{t}$ and the probability of emission $P$.

\section{TRANSMISSION GAIN}

The transmission gain is defined as the ratio of the current of the secondary electrons reaching the emission surface to the primary-electron current. In our earlier transmission-mode experiment, we terminated both diamond surfaces with metal. Then, the measured transmission gain depends only on the primary electrons' energy and diamond's internal electric field [2]. The model of secondary-electron generation and transmission in the diamond are discussed in Ref. [3]. However, the agreement with transmission experimental data is generally qualitative 


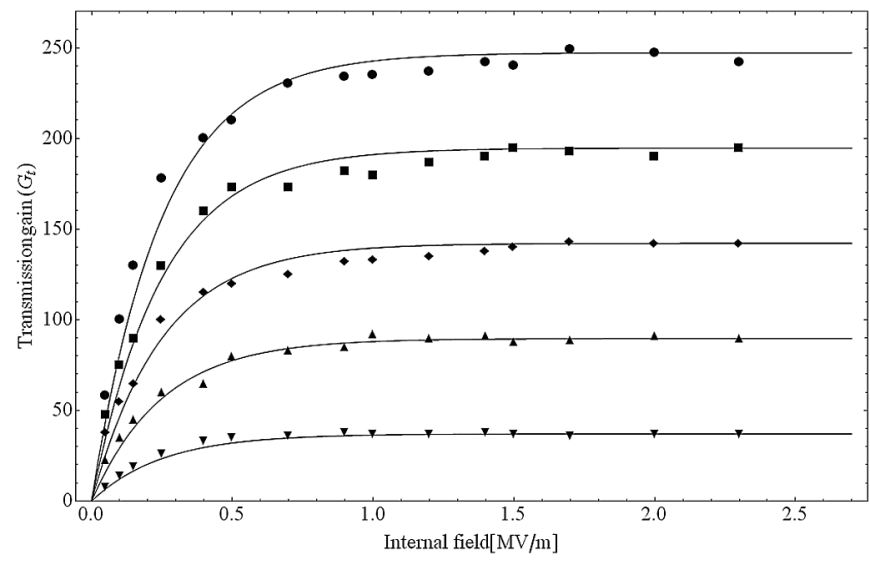

FIG. 2. Transmission gain $\left(G_{t}\right)$ curve of a synthetic high-purity single crystalline diamond. The points are the experiment results (reproduced from Ref. [3] with permission of authors) and thin solid lines are the fitting function. The six lines are a response to the different primary beam energies.

and not quantitative. In this paper, we find that the gain fits rather well to an easy functional dependence which includes the primary electron's energy and the field in the diamond as follows:

$$
G_{t}\left[\mathcal{E}_{p}, E_{i}(t)\right]=\left(a \mathcal{E}_{p}-b\right)\left(1-e^{-c E_{i}(t)}\right),
$$

where $\mathcal{E}_{p}$ is the primary electron's energy, and $E_{i}(t)$ is the time-dependent internal field. The points in Fig. 2 show transmission gain as a function of internal field measured for a diamond sample. The combined best fit of Eq. (6) to the data, shown as solid lines in Fig. 2, yields $a=$ $52.5 \mathrm{keV}^{-1}, b=173.2$, and $c=4.1(\mathrm{MV} / \mathrm{m})^{-1}$. If the curves are fitted individually, the coefficients $a$ and $b$ are the same as before and $c$ is in the range of 4.1 to 4.4 for different primary electron energy but still within the experiment error. Therefore, we use the coefficients from the combined best fit in the following simulations.

\section{DERIVATION OF EMISSION PROBABILITY}

Next, we obtained the emission probability using the Schottky model applied to the negative-electron affinity (NEA) of the hydrogenated diamond's surface. There are two ways to model the NEA phenomenon; either in terms of true negative-electron affinity or of effective negative affinity. The latter model is described as a combination of positive electron affinity and the bending of the depletion band at the surface of the semiconductor. For true NEA, the vacuum level is lower than the minimum of the conduction band at the surface. Therefore, an electron at the conduction band minimum $(\mathrm{CBM})$ is free to leave the crystal because the barrier does not exist. In our experiment (measurements given below), we found that electrons are trapped at the emission surface, and furthermore, that the trapping rate is a function of the external field. The potential surface barrier prevents electrons with energy below this barrier from escaping the diamond and the modification of this barrier by the applied field changes the number of electrons that can escape, thus leading to a measurement of the electrons' energy distribution. This is in agreement with diamond $(001)-(2 \times 1): \mathrm{H}$ surface structure calculation in other work [4].

We used four practically identical single-crystal, high-purity chemical vapor-deposition diamonds [100] to fabricate four diamond amplifiers by applying a thin metal layer on one surface, and hydrogen termination of the other surface. The preparation of the amplifiers is detailed elsewhere [1].

We carried out the experiment by measuring the emission current as a function of the pulse's length and the strength of the applied field. Figure 3 shows the $G_{e} / G_{t}$ of four diamonds as a function of the external field where we used a very small pulse width (200 ns). Under this very short pulse, and the primary current that we applied (200 nA), the change in the internal field can be neglected, and the instantaneous emission probability, $P$, is equal to $G_{e} / G_{t}$.

We adopted the Schottky effect on the effective NEA surface to explain why emission probability depends on the external field. The diamond crystal is a dielectric. The external electric field and the force from image charges inside the diamond reduce the electron's potential energy according to the following expression [5]:

$$
V(x)=-\frac{e^{2}}{16 \pi \varepsilon_{0}}\left(\frac{\varepsilon-\varepsilon_{0}}{\varepsilon+\varepsilon_{0}}\right) \frac{1}{x}-e E_{e} x,
$$

where $x$ is the distance from the emission surface, $\varepsilon$ is the permittivity of the diamond, and $\varepsilon_{0}$ is the permittivity of the vacuum. We compute the maximum value of the potential by setting $d V(x) / d x$ to zero. The difference between the maximum value of the potential and the vacuum level then is given by

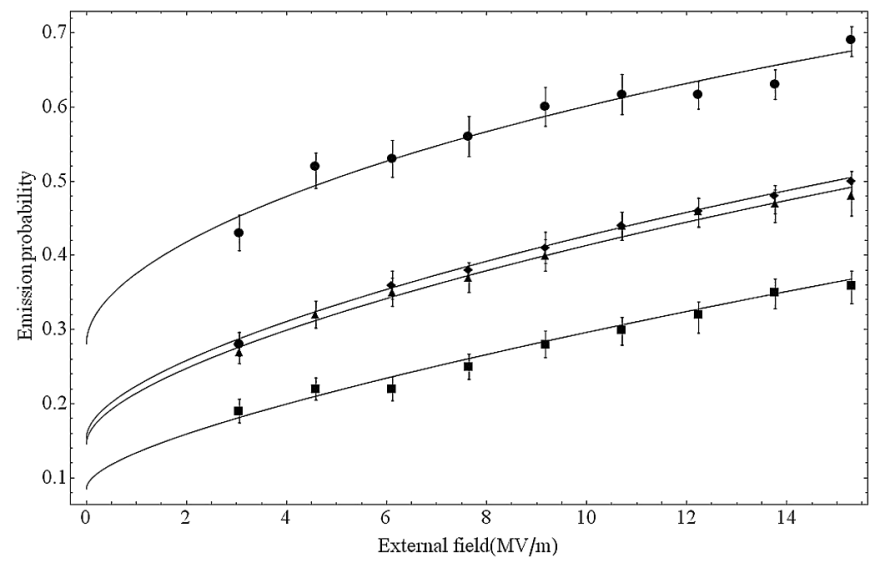

FIG. 3. The dependence of emission probability $P$ on the external field when the pulse width is $200 \mathrm{~ns}$. The points were measured from four different diamond samples. The four solid lines were generated by fitting to Eq. (11), below. 


$$
\phi_{s}=\sqrt{\frac{e E_{e}}{4 \pi \varepsilon_{0}}\left(\frac{\varepsilon-\varepsilon_{0}}{\varepsilon+\varepsilon_{0}}\right)} .
$$

The diamond's relative permittivity is 5.6 , the Schottky potential simplifies to $0.0318\left(E_{e}(\mathrm{MV} / \mathrm{m})\right)^{0.5}[\mathrm{eV}]$. The Schottky effect reduces the surface potential barrier, thus allowing the emission of electrons with a lower energy. Figure 4 shows the diamond surface's band structure. As secondary electrons reach the emission surface, some get into the potential well between the conduction band and the Schottky potential. The minimum energy of the secondary electron is the same as that of the CBM at the surface. We define $\varphi_{1}$ as the energy difference from the CBM to the vacuum level. The $\varphi_{1}$ reduce in the potential $\varphi_{S}$ due to Schottky effect. Electrons can escape the diamond either with energies great than $\varphi_{1}-\varphi_{S}$, or by tunneling through the barrier.

We find that fitting the current dependence on the applied field to an expression [6] used for tunneling does not lead to a good fit to the experimental data. On the other hand, we get a good fit making the assumption that only electrons above the Schottky barrier escape and neglecting tunneling altogether.

The emission probability also is related to the energy distribution of electrons inside the diamond. This distribution depends on the electron-transport process in the bulk. On the other hand, the energy distribution of the electrons emitted from a surface depends strongly on the position of the vacuum level and the Schottky potential, which are related to the external field.

As a first approximation, we model the distribution of secondary electrons in diamond near the emission surface (close to the end of the band-bending region) with a Gaussian given by

$$
f(\varphi)=\frac{e^{-\left[(\varphi-m)^{2} / 2 \sigma^{2}\right]}}{\sqrt{2 \pi \sigma^{2}}},
$$



FIG. 4. Energy-level diagram of diamond amplifier/vacuum interface band structure. The dashed curve represents the internal distribution of the secondary electrons' energy. $\varphi_{1}$ is the energy difference from the CBM to the vacuum level and $\varphi_{m}$ is the energy difference between the mean of distribution of internal electron energy and the vacuum level. $\varphi_{S}$ is the reduction of the barrier by the applied field. where $\sigma$ is the variance, and we chose the mean as $m$ relative to $\mathrm{CBM}$ at the surface. $\varphi$ is taken as the energy above the CBM. For the electrons with energy lower than $\varphi_{1}-\varphi_{s}$, the probability of escape is assumed negligible.

Therefore, the probability of secondary-electron emission is

$$
P=1-\int_{-\infty}^{\varphi_{1}-\varphi_{s}} f(\varphi) d \varphi
$$

Combining Eqs. (8)-(10) generates a simple expression for the emission probability in terms of the width of the energy distribution, the applied electric field, and the NEA:

$$
P=1-\frac{1}{2} \operatorname{Erfc}\left(\frac{\varphi_{m}+\varphi_{s}}{\sqrt{2} \sigma}\right)
$$

where $\varphi_{m}$ is the energy difference between the mean distribution of internal electron energy and the vacuum level. The values of $\sigma$ and $\varphi_{m}$ now are found by fitting to the experimental data (Fig. 2).

Since all four diamond samples were of the same thickness, crystal orientation, and purity, we assume that the internal distribution of secondary electrons is the same in all of them under the same measurement conditions. However, the level of the NEA may differ among these samples, as is reflected by changes in $\varphi_{m}$. The internal energy spread, $\sigma$, obtained from the best fit is $0.12 \pm$ $0.01 \mathrm{eV}$, in agreement with simulation showing that $\sigma$ is 0.13 to $0.14 \mathrm{eV}$ [7]. For the four diamonds, the values of $\varphi_{m}$ obtained from the fitting are $-0.070,-0.123,-0.127$, and $-0.165 \mathrm{eV}$. The vacuum level of the different samples might vary due to several effects, such as hydrogen coverage [8], surface-carbon orientation [9,10], and the orientation of the $\mathrm{C}-\mathrm{H}$ bond in the hydrogen-terminated surface [11]. Equation (11) gives the initial emission-probability response to the external field with a certain $\sigma$ and $\varphi_{m}$, regardless of how it is determined.

The band structure calculations of Watanabe et al. [12] show that for applied fields lower than $10 \mathrm{MV} / \mathrm{m}$, the normalized energy distribution per unit energy divided by the density of states (DOS) behaves as a non-normalized Boltzmann distribution with effective temperature [Fig. 7(a) in their paper]. However, their results are for electron transport in bulk diamond and do not take into account how the band-bending region affects the distribution of electrons as a function of energy. We considered fitting the observed simulation data for the number of electrons per unit energy with a Boltzmann distribution times a model DOS given by $\sqrt{\mathcal{E}}$ but a better fit was obtained using a simple Gaussian distribution. A more detailed theoretical model is needed to obtain a better understanding of the energy distribution of electrons near the emission surface that also takes into account the bandbending effects. 
TABLE I. The initial parameters for solving integral Eq. (5).

\begin{tabular}{lc}
\hline \hline Spot size & $0.55 \mathrm{~mm}^{2}$ \\
Voltage between metal coating and anode & $3000,5000 \mathrm{~V}$ \\
Frequency & $1000 \mathrm{~Hz}$ \\
Diamond thickness $\left(L_{d}\right)$ & $300 \mu \mathrm{m}$ \\
Anode gap $\left(L_{g}\right)$ & $250 \mu \mathrm{m}$ \\
$\varphi_{m}$ & $-0.07 \mathrm{eV}$ \\
Primary current $(\mathrm{DC})$ & $200 \mathrm{nA}$ \\
\hline \hline
\end{tabular}

\section{SOLVING THE INTEGRAL EQUATION (5)}

Now that we have established the emission probability, Eq. (5) can be solved numerically to obtain the pulselength dependence of the emission current. The initial conditions we used in solving the equation are listed in Table I.

The measurable parameter in the experiment is the average secondary-electron emission current that engenders the average emission gain $G_{e}$. We obtain the average emission current by integrating the emission current over the pulse's width, and dividing this by the period:


$$
I_{a}=\frac{\int_{0}^{w} I_{p} \times G_{t}\left[\mathcal{E}_{p}, E_{i}(t)\right] \times P d t}{1 / f} .
$$

We now insert the emission probability, Eq. (11), and the transmission gain, Eq. (6), into the integral, Eq. (5). The integral equation is solved numerically to generate the internal electric field as a function of time along the pulse length. Once this is known, average emission current and average emission gain can be calculated. Figures 5(a)-5(d) illustrate our results.

We measured the sample wherein $\varphi_{m}$ is $-0.07 \mathrm{eV}$ based on the initial conditions shown in Table I. The setup for measuring the secondary current is published elsewhere [13]. The normalized values for the secondary-electron emission gain, indicated by the points in Fig. 5, match well the model's prediction.

The process leading to this behavior is a decrease of the internal field along the pulse's width due to the accumulation of trapped electrons on the surface and thus shielding of the external field. As the internal field decreases, the secondary electrons drift more slowly towards the emission surface and incur further recombination loss at the surface
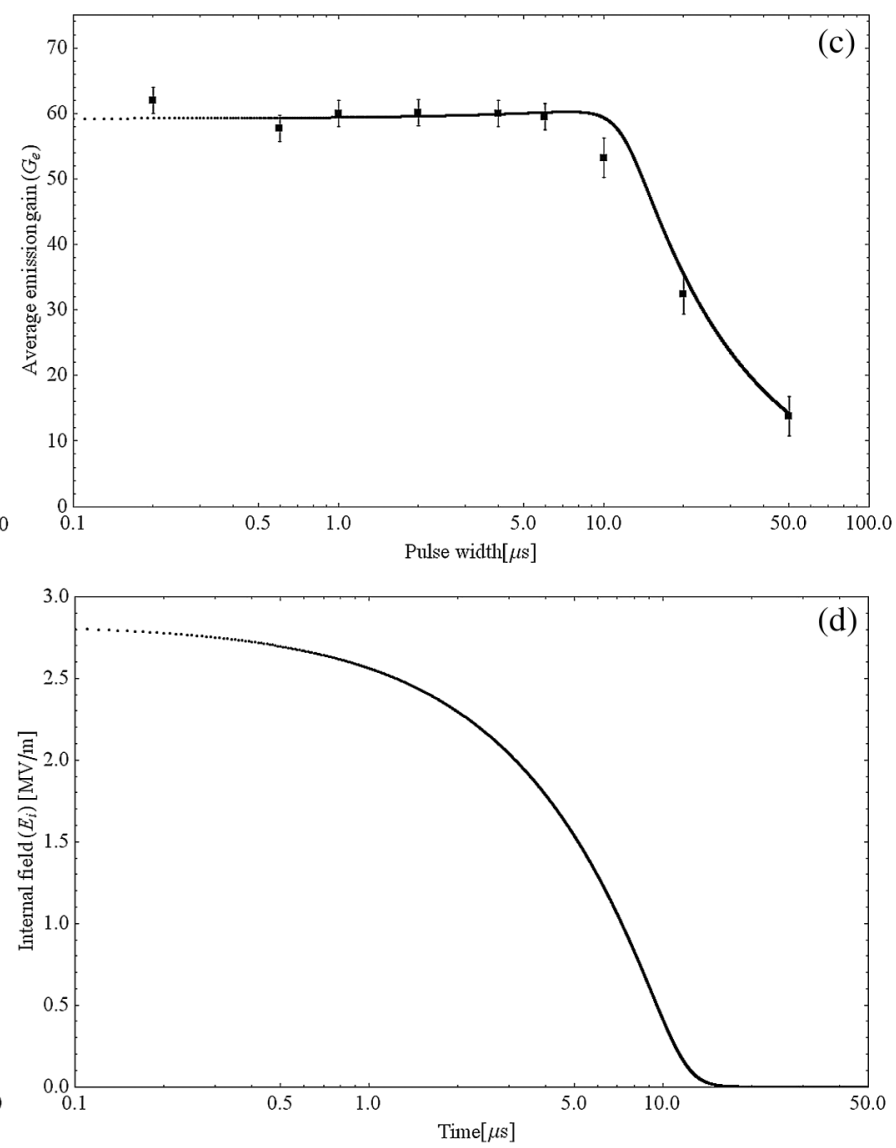

FIG. 5. The results of resolving Eq. (5). Parts (a) and (b), respectively, are the average emission gain $\left(G_{e}\right)$, and the time dependence of internal field $\left(E_{i}\right)$ for an applied voltage of $3000 \mathrm{~V}$. Parts (c) and (d), respectively, are the average emission gain $\left(G_{e}\right)$, and the time dependence of internal field $\left(E_{i}\right)$ for an applied voltage of $5000 \mathrm{~V}$. The solid squares are the experimental results accompanied by the estimated systematic error bars. The continuous curves are the solution of Eq. (5). 
of the metal layer; therefore, the average emission gain and normalized emission rate decrease.

\section{THE ENERGY SPREAD DUE TO THE IMFP}

The electron's energy spread near the emission surface is not determined by the energy of the nascent electrons; in drifting through the diamond, the electrons undergo a vast number of collisions, both elastic and inelastic. The energy spread of the electrons is the product of equilibrium between the small energy gain during their transit from the internal field, and their energy loss due to the frequent scattering they experience. The inelastic mean-free path (IMFP) is energy dependent; thus, the electric field affects the equilibrium temperature attained by the drifting electrons. The equilibrium value of random energy as a function of the inelastic mean-free path is estimated as follows [14]:

$$
\frac{1}{2} m V_{e}^{3}=-e E v \lambda_{i}
$$

where $V_{e}, m, v$, and $\lambda i$ are, respectively, the electron's velocity, electron's effective mass, the electron's drift velocity, and IMFP. The drift velocity is expressed by $v=10^{5} \times\left(0.2 E_{i}[\mathrm{MV} / \mathrm{m}]+0.55\right)[\mathrm{m} / \mathrm{s}]$ For the IMFP, we use the semiempirical formula of Seah and Dench [15]:

$$
\lambda_{i}=\left[538 \mathcal{E}_{r}^{-2}+0.41\left(x_{m} E_{r}\right)^{1 / 2}\right] x_{m} .
$$

The $x_{m}$ is the thickness of a monolayer in nanometers that, for diamonds, is $0.178 \mathrm{~nm}$. $\mathcal{E}_{r}$ is the electrons' energy above the Fermi level and the unit is eV. For the intrinsic diamond, the energy of the conduction band above the Fermi energy is $2.775 \mathrm{eV}$. By inserting Eq. (14) into Eq. (13), the equilibrium electron random energy is $0.04 \mathrm{eV}$, and IMFP is $12 \mathrm{~nm}$ when the internal field is $2 \mathrm{MV} / \mathrm{m}$. This calculated random energy of the electron is much smaller than the measured width $\sigma$ of the Gaussian distribution above the conduction band obtained from the Schottky model. Since the Seah and Dench model does not consider the band-bending region at the diamond's surface, the physics of the electron motion in this region offers an explanation for the discrepancy. The depth of the band-bending region was estimated from experiments to be about $0.1 \mu \mathrm{m}[7,10]$, viz., several times larger than the IMFP. The electrons scatter with phonons and emit acoustic phonons several times in the varying energy level at the band-bending region. Meanwhile, since part of the electrons' energy extends above $0.13 \mathrm{eV}$ [12], optical phonon emission takes place in the band-bending region. We propose this scattering to be responsible for the increase in the electrons' energy spread inside the diamond and consider that the energy spread may be reduced by a modification of the length of the band-bending region.

\section{CONCLUSIONS}

We measured the emission probability of the diamond amplifier as a function of the external field and modeled the process with the resulting changes in the vacuum level due to the Schottky effect. The effective NEA is deduced from the model and measurements. We demonstrated that the average decrease in the secondary electrons' emission gain was a function of the pulse width, and related this to the trapping of electrons by the effective NEA surface. Based on our measurement of four diamond samples with different effective NEAs (but otherwise identical), we obtained the distribution of the secondary-electrons' internal energy, $\sigma$, which was $0.12-0.01 \mathrm{eV}$ in all four samples. This energy spread is in agreement with the simulation by Dimitrov et al. [7], but is larger than the assessment made by Seah and Dench [15]. We attribute this difference to electron scattering in the band-bending region next to the diamond's surface.

The emission model currently implemented in the VORPAL code [7] considers only a stair-step potential at the emission surface. This is the simplest possible model for emission (and also the least complex to implement in the code). The results reported in [7] were with the stairstep model. The main deficiency of this model is that it does not take into account the effect of the applied field on the surface potential and thus is not expected to be accurate. We are currently exploring to add a more realistic surface potential in the simulation code and fully take into account the effect of applied field during emission.

Compared to the earlier model, there are three significant advances in this new model: (i) The emission model derived by the VORPAL computational framework did not consider the surface electron trapping which is described well in the model of this paper. (ii) We observed experimentally that the emission probability depends on the external field. We introduce the Schottky effect on the effective NEA surface to explain this phenomenon, which cannot be explained by the model run in VORPAL [7]. (iii) Some parameters such as $\varphi_{m}$ and electron energy distribution of this emission model can be fitted by the experimental data from four diamond samples.

\section{ACKNOWLEDGMENTS}

The authors acknowledge Dr. David H. Dowell for useful discussions and suggestions. This work was carried out at Brookhaven Science Associates, LLC under Contract No. DE-AC02-98CH10886 and at Stony Brook University under Grant No. DE-SC0005713 with the U.S. DOE.

[1] E. Wang et al., Phys. Rev. ST Accel. Beams 14, 061302 (2011).

[2] X. Chang et al., Phys. Rev. Lett. 105, 164801 (2010).

[3] D. A. Dimitrov et al., J. Appl. Phys. 108, 073712 (2010).

[4] S. J. Sque, R. Jones, and P. R. Briddon, Phys. Rev. B 73, 085313 (2006). 
[5] J. Jackson, Classical Electrodynamics (Wiley, New York, 1998), 3rd ed.

[6] R. H. Fowler and L. Nordheim, Proc. R. Soc. London 119, 173 (1928).

[7] D. Dimitrov, R. Busby, and D. Mithe, in Proceedings of 2011 Particle Accelerator Conference, New York, WEP161 (to be published).

[8] I. L. Krainsky and V. M. Asnin, Appl. Phys. Lett. 72, 2574 (1998).

[9] J. Furthmuller, J. Hafner, and G. Kresse, Phys. Rev. B 53, 7334 (1996).

[10] F. Maier, J. Ristein, and L. Ley, Phys. Rev. B 64, 165411 (2001).
[11] Z. Zhang, M. Wensell, and J. Bernholc, Phys. Rev. B 51, 5291 (1995).

[12] T. Watanabe et al., J. Appl. Phys. 95, 4866 (2004).

[13] X. Chang et al., in Proceedings of the 23rd Particle Accelerator Conference, Vancouver, Canada, 2009 (IEEE, Piscataway, NJ, 2009).

[14] T. Rao et al., in Diamond Amplifier for Photocathodes, AIP Conf. Proc. No. 737 (AIP, New York, 2004), p. 178.

[15] M.P. Seah and W.A. Dench, Quantitative Electron Spectroscopy of Surfaces: A Standard Data Base for Electron Inelastic Mean Free Paths in Solids (Heyden and Son Ltd., London, 1979), pp. 2-11. 